\title{
VIII. „Wiedergutmachung“
}

Zählten zu den Opfern des Dritten Reiches neben Millionen Ausländern auch eine große Zahl von Angehörigen des eigenen Staates aus der jüdischen Bevölkerungsgruppe oder aus dem Widerstand, so waren vom japanischen Ultranationalismus in allererster Linie Menschen fremder Länder an Leib und Leben geschädigt worden. Deshalb war die für die bundesrepublikanische „Wiedergutmachung "1 charakteristische Mischung aus außen- und innenpolitischen Bezügen in Nippon so nicht anzutreffen, sondern stellte sich die Entschädigung sehr viel eindeutiger als ein Problem der internationalen Beziehungen dar. Einheimische koreanische oder taiwanesische Kriegsopfer aus den ehemaligen Kolonien, die nach der Kapitulation 1945 die japanische Staatsbürgerschaft verloren hatten, wurden in dem 1952 erlassenen Gesetz zur Unterstützung der (japanischen) Kriegsopfer und ihrer Angehörigen mittels einer Nationalitätsklausel von vornherein aus dem Kreis der Anspruchsberechtigten ausgegrenzt.

Als noch problematischer erwies sich, daß von der Friedenskonferenz in San Francisco 1951 ausgerechnet Vertreter des chinesischen Volkes, das am meisten unter der japanischen Kriegführung gelitten hatte und die größten Entschädigungsansprüche besaß, ausgeschlossen blieben. Dazu geführt hatten Meinungsverschiedenheiten zwischen Großbritannien, das die Volksrepublik China anerkannt hatte, und den USA, die zwischenzeitlich auf das nationalchinesische Regime des nach Taiwan geflohenen Jiang Kaishek setz$\operatorname{ten}^{2}$. So mußten die Regierungen Nationalchinas und Japans 1952, von den USA auf einem Höhepunkt des Koreakonflikts zur Einigung gedrängt, einen bilateralen Friedensvertrag schließen, der für die chinesische Seite ausgesprochen ungünstig ausfiel. Als Taiwan Reparationen auch für das chinesische Festland als eine Frage der nationalen Würde auf die Agenda brachte, zog Japan die Zuständigkeit des Jiang-Kaishek-Regimes für Gesamtchina in Zweifel. Am wundesten Punkt getroffen gab Taipeh nach und erklärte im Abschlußprotokoll: „Als Ausdruck seiner Großzügigkeit und seines guten Willens gegenüber dem japanischen Volk verzichtet [Taiwan] aus eigenem

1 Zur bekannten Problematik des Begriffes vgl. am besten die abwägende Darstellung von Hockerts, Wiedergutmachung, S. $167 \mathrm{ff}$.

2 Zum Verhältnis britischer und amerikanischer Japanpolitik Buckley, Occupation Diplomacy; speziell zum Ringen um den Friedensvertrag, S. 159-183. Hierzu auch Yoshitsu, Japan and the San Francisco Peace Settlement. 
Antrieb auf die Erträge der von Japan zu erbringenden Dienstleistungen“"3. Für Tokio waren damit sämtliche im Zusammenhang mit dem Krieg stehende Fragen zwischen Japan und China abschließend geklärt. Schuldgefühle gegenüber dem von allen Nachbarn historisch am engsten mit Japan verbundenen Land kamen lediglich bei wenigen Intellektuellen auf ${ }^{4}$.

Die Verdrängung des dunkelsten Kapitels in der Geschichte der beiderseitigen Beziehungen wurde auf der anderen Seite auch dadurch begünstigt, daß die Regierung in Peking selbst etwa die Veröffentlichung eines Manuskripts unterband, das Historiker an der Universität Nanking 1962 vorgelegt hatten. Rotchina hatte sich eben erst vom jüngsten ausländischen Einfluß befreit - dem der Sowjetunion, die das große Nachbarland ihrem Machtblock so weit wie möglich einzuverleiben trachtete. Ziel der Politik Pekings war es infolgedessen, die chinesische Bevölkerung mit Stolz auf ihre Nation zu erfüllen, statt den Fokus der Aufmerksamkeit auf eine Zeit der nationalen Schwäche zurückzulenken ${ }^{5}$. Hinzu kam der marxistischer Ideologie geschuldete Wille, die Geschichte Chinas vor 1949 mit der Brille des Klassenkampfes zu betrachten, wobei vor allem die unter der nationalistischen Regierung herrschenden Klassen der Großgrundbesitzer und Kapitalisten das - innerchinesische - Feindbild abgaben, während nationale Konflikte mit ausländischen Mächten demgegenüber eher zweitrangig waren'

China stellt insofern einen Sonderfall dar, als Japan mit den meisten übrigen Hauptgeschädigten des Ostasiatisch-Pazifischen Krieges zumindest in der einen oder anderen Art Entschädigungsabkommen traf. Grundlage dessen war der von amerikanischer Seite mit großer Nachsicht gegenüber Japan ausgehandelte Friedensvertrag von San Francisco, der in Artikel 14 zwar festhielt, daß das Land „den Alliierten für die im Krieg verursachten Schäden und Leiden Reparationen zu zahlen hat", die Verpflichtung jedoch zugleich dadurch relativierte, daß, „wenn man davon ausgeht, daß eine existenzfähige Wirtschaft aufrecht zu erhalten ist, Japans Ressourcen derzeit nicht ausreichen, um Reparationen für die oben erwähnten Schäden und Leiden vollständig zu leisten und gleichzeitig andere Schulden zurückzuzahlen"7.

Die ersten, bereits 1951/52 erhobenen Forderungen Indonesiens und der Philippinen in Höhe von 18 bzw. 8 Billionen US-Dollar konnte Japan vor

Zit. nach Tanaka, Japans Nachkriegsverantwortung, S. 401.

Olson, Japan in Postwar Asia, S. 100.

5 Eykholt, Chinese Historiography, S. 25.

6 Yang, The Malleable and the Contested, S. 54.

7 Zit. nach Tanaka, Japans Nachkriegsverantwortung, S. 398. Zur Bedeutung von San Francisco für die japanischen Reparationen vgl. auch Seiffert, Die Reparationen Japans, S. $129 \mathrm{ff}$. Die Studie orientiert sich im übrigen vor allem an der Fragestellung internationaler technisch-wirtschaftlicher Zusammenarbeit, ohne die - sogenannten - „psychologischen“ Probleme der „Vergangenheitsbewältigung" zu vertiefen, und kommt infolgedessen zu einem ausgesprochen positiven Befund der japanischen Entschädigungspolitik. 
diesem Hintergrund leicht zurückweisen, entsprachen doch bereits die 8 Billionen ungefähr dem dreifachen des japanischen Haushalts im Jahr 1954. Erst als die innerparteiliche Opposition gegen den langjährigen konservativen Premierminister Yoshida den Fehlschlag der bisherigen Reparationsverhandlungen als Argument gegen ihn einsetzte und in dieser Situation im Sommer 1954 eine birmesische Delegation nach Tokio reiste, kam Bewegung in die Sache. Im Frühjahr 1955 konnte eine Vereinbarung über Reparationen und reparationsähnliche Zahlungen in Höhe von 250 Millionen Dollar ratifiziert werden ${ }^{8}$.

Yoshidas Nachfolger setzten nun verstärkt auf ökonomische Kooperation mit Südostasien, um damit die Grundlagen für eine politische Stabilisierung der Region zu verbessern. Spätestens auf der afro-asiatischen Konferenz in Bandung, wo die blockfreien Staaten im April 1955 erstmals versuchten, das weltpolitische Gewicht der Dritten Welt zu erhöhen, wurden die dort hingereisten Politiker und Wirtschaftsführer aus Japan in der Auffassung bestärkt, daß eine zu ausschließliche Anlehnung an die USA sie als „gelbe Amerikaner" $"$ verdächtig machte, daß es mithin galt, auch eigene Anstrengungen zu unternehmen, um den während des Weltkrieges angerichteten außenpolitischen Scherbenhaufen zu beseitigen und nicht als „Neokolonialisten“ $\mathrm{zu}$ erscheinen.

Einen wichtigen Schritt in diese Richtung markierte im Sommer 1956 das Reparationsabkommen mit den Philippinen, wo die antijapanische Stimmung aufgrund der Ereignisse während des Krieges mit am stärksten war. Vereinbart wurden Reparationsgüter in Höhe von 500 Millionen Dollar über einen Zeitraum von 20 Jahren, dazu technische Dienstleistungen im Wert von 30 Millionen sowie 20 Millionen für Kriegswitwen und -waisen. Auf den Vertrag mit den Philippinen, an die insgesamt die mit Abstand größte Summe gezahlt wurde, folgten in den 1950er Jahren noch Abkommen mit Indonesien (1958) und mit einigen weniger stark geschädigten Ländern wie Laos (1958), Kambodscha (1959) und Südvietnam (1959). In der zweiten Hälfte der 1960er Jahre kamen weitere kleinere Staaten wie Malaysia und Singapur hinzu. Von wenigen Ausnahmen abgesehen wurden die Kriegsopfer außerhalb Japans also nicht individuell entschädigt, sondern nur indirekt durch Globalbeträge an die Regierungen und den Verzicht auf japanische Vermögen im Ausland. Die dabei geleistete Wirtschaftshilfe wie z. B. der Bau von Staudämmen oder Eisenhüttenwerken kam obendrein der wirtschaftlichen Expansion Japans in die asiatischen Nachbarländer zugute.

Als besonders schwierig erwies sich die vertragliche Regelung mit Südkorea, die erst nach langem Ringen 1965 zustande kam. Südkorea war zwar (ebenso wie Nordkorea) nicht zur Friedenskonferenz nach San Francisco

9 Ebd., S. 24. 
eingeladen worden und konnte bei den USA auch kaum Unterstützung für seine Reparationsforderungen an Japan (wie die Rückgabe aller Goldreserven und Kunstgüter oder die Zahlung ausstehender Löhne für koreanische Zwangsarbeiter) finden, die mittelbar praktisch vom amerikanischen Steuerzahler hätten geleistet werden müssen. Allerdings lag den USA viel an einer Klärung der - durch die sogenannte MacArthur-Linie nur provisorisch geregelten - hochbrisanten maritimen Grenz- bzw. Fischereifragen zwischen seinen beiden engsten Klienten in der Region, so daß sie der Aufnahme separater Verhandlungen zwischen Tokio und Seoul noch im Herbst 1951 zustimmten. Weitere Streitpunkte waren die Rechtsstellung der in Japan verbliebenen 600000 Koreaner und die Frage, ob der Vertrag von 1910 über die Annexion Koreas durch Japan ex tunc oder nur ex nunc als ungültig anzusehen sei. Als die Verhandlungen nicht vorankamen, ja die Spannungen nach der Verhaftung von über tausend japanischen Fischern durch Südkorea eskalierten, bemühten sich die USA um das Zustandekommen eines inoffiziellen Treffens zwischen Premier Yoshida und dem südkoreanischen Präsidenten Syngman Rhee im Januar 1953, das aber nur die Tiefe des Grabens zwischen den Nachbarländern und deren Hauptursache, das mangelnde Schuldbewußtsein der Japaner, demonstrierte. Rhee, im Guerillakrieg gegen die japanische Besatzungsmacht sozialisiert und zu einem leidenschaftlichen Nationalisten geworden, verlangte von Yoshida eine Entschuldigung für die japanische Kolonialherrschaft, stieß bei diesem aber auf fast völlig taube Ohren ${ }^{10}$.

Schob Yoshida die Verantwortung für die ultranationalistische Vergangenheit im Gespräch mit Rhee eher defensiv einfach auf „die Militärcliquen" ab, so enthüllte der japanische Delegationsführer bei einer weiteren Verhandlungsrunde im Oktober 1953, Kubota Kan 'ichirô, ohne jede diplomatische Schminke den Standpunkt Tokios und breitete ein Panorama der kolonialen Wohltaten aus: Japan habe in Korea die kahlen Hügel aufgeforstet, Bewässerungsanlagen errichtet und dadurch die Reisproduktion erhöht, Eisenbahnen, Straßen und Schulen gebaut, also insgesamt gewaltige Gelder in Korea investiert. Um den Pelion auf den Ossa zu türmen, behauptete Kubota auch noch, die alliierten Deklarationen von Kairo und Potsdam über die „Versklavung des koreanischen Volkes“ seien unbedacht, „in der Hitze des Gefechts" 11 formuliert worden, und wiederholte dies - um an der Ernsthaftigkeit seiner Thesen keinen Zweifel aufkommen zu lassen - wenig später noch einmal im Oberhaus. Tatsächlich hatte Kubota nur einer in der japanischen Bevölkerung weit verbreiten Auffassung Ausdruck verliehen, die etwa auch der Gouverneur von Nagasaki 1955 in die Frage kleidete:

$10 \mathrm{Vgl}$. Fuhrt, Erzwungene Reue, S. 51-54, sowie Olson, Japan in Postwar Asia, S. 103.

11 Fuhrt, Erzwungene Reue, S. 55. 
„Warum können die Koreaner Japan gegenüber nicht so empfinden wie die Inder gegenüber Britannien?" 12

Infolgedessen stand Kubota, als in den parlamentarischen Gremien über den durch seine Äußerungen verursachten Abbruch der Gespräche debattiert wurde, unter keinem nennenswerten Rechtfertigungsdruck. Lediglich ein sozialistischer Abgeordneter warf ihm vor, mit seinen Aussagen das diktatorische Regime des südkoreanischen Präsidenten Rhee zu stützen. Ansonsten überwog auch im Blätterwald eindeutig die Kritik an dem „allzu emotionalen Verhalten“ der koreanischen Verhandlungsdelegation. Auch die US-Regierung machte - nicht zuletzt unter dem Einfluß ihres Botschafters in Tokio - deutlich, daß sie in den antijapanischen Ressentiments des Rhee-Regimes die Hauptursache für die Spannungen zwischen Seoul und Tokio erblickte ${ }^{13}$. Zweifelsohne war diese Einschätzung aber auch dadurch beeinflußt, daß gerade damals Japan für die USA der mit Abstand wichtigste Partner in der Region war. Im Sommer 1954 begann Tokio nach dem Abschluß eines japanisch-amerikanischen Abkommens über gegenseitige Verteidigungshilfe mit dem Aufbau moderner Land-, See- und Luftstreitkräfte („Selbstverteidigungsstreitkräfte“). Spätere Versuche der USA, zwischen Japan und Südkorea zu vermitteln, schlugen zunächst sämtlich fehl, obwohl sich Japan im Frühjahr 1958 immerhin zur Rückgabe von 106 Kulturgütern an Seoul durchrang, allerdings ausdrücklich nicht - wie der Leiter des Tokioter Asienreferates nach Bekanntwerden der Geheimaktion in einer Fragestunde vor dem Oberhaus darlegte - „als geraubte Beutekunst“, sondern lediglich „zur Verbesserung der Atmosphäre“ zwischen beiden Ländern14.

Erst nach dem Sturz Rhees 1960 setzte unter der demokratischen Übergangsregierung und ab 1961 unter der Militärjunta General Chung-hee Parks ein Wandel in den Beziehungen beider Länder ein ${ }^{15}$. Darauf drängten zum einen die USA angesichts eigener wirtschaftlicher Schwierigkeiten und der sich zuspitzenden Situation in Indochina. Und auch in Südkorea und Japan selbst versprach man sich zunehmend ökonomische Vorteile von einer Einigung. Während aber in Südkorea auch verändertes Denken bei einer neuen politischen Elite Einzug hielt, die sich während der Kolonialzeit überwiegend mit Japan arrangiert hatte und mit dessen Kultur vertraut war, spielte bei den japanischen Verfechtern einer Annäherungspolitik ein $\mathrm{Be}$ wußtseinswandel hinsichtlich der Vergangenheit keine nennenswerte Rolle. Shiina Etsusaburô, während der entscheidenden Verhandlungsphase 1964/65 japanischer Außenminister, hatte erst in seinen 1963 erschienenen Erinnerungen an die Kriegszeit einen bemerkenswerten Einblick in sein Geschichtsbild gestattet: „Wenn man die Tatsache, daß Japan, um Asien zu

12 Olson, Japan in Postwar Asia, S. 109, sowic Paik, Korea und Japan, S. 183-188.

13 Allison, Ambassador from the Prairie, S. 258f., sowie Fuhrt, Erzwungene Reue, S. $56 \mathrm{ff}$.

14 Fuhrt, Erzwungene Reue, S. 61.

15 Zum folgenden die instruktive Analyse bei Fuhrt, Erzwungene Reue S. 63-76. 
schützen und seine eigene Unabhängigkeit zu bewahren, seit der Meiji-Zeit Taiwan bewirtschaftete, Korea annektierte und in der Mandschurei auf den Traum von der Harmonie der fünf Völker setzte, als japanischen Imperialismus bezeichnet, dann war das ein ruhmreicher Imperialismus."16

Nicht etwa historische Schuldgefühle bestimmten die japanische Koreapolitik, sondern das nüchterne Kalkül, mit der Militärdiktatur Parks, die auf die antijapanische Öffentlichkeit des Landes weniger Rücksicht zu nehmen brauchte, am ehesten eine Normalisierung zu einem für Japan möglichst niedrigen Preis zu erreichen. Schließlich konnte man davon ausgehen, daß das Interesse Südkoreas an ökonomischer Zusammenarbeit mit Japan nach dem Verlust der Bodenschätze im Norden dringender war als umgekehrt. Tatsächlich erklärte sich Park, als er auf dem Weg nach Washington in Tokio Zwischenstation machte, bereit, die koreanischen Ansprüche nicht durch explizite Entschädigung für die japanische Kolonialherrschaft zu regeln, sondern auf dem Wege einfacher Wirtschaftshilfe. Laufende Expertengespräche über individuelle Ansprüche von 77000 im japanischen Militär- und Arbeitsdienst umgekommenen Koreanern sowie 25000 Versehrten wurden durch die Einigung gegenstandslos. Zumindest aber mußte sich Tokio, nicht zuletzt auf amerikanisches Drängen hin, zu einer Erklärung durchringen, die Minimalforderungen Koreas hinsichtlich einer Distanzierung von der Kolonialherrschaft erfüllte. In einer kurzen Ansprache bei seiner Ankunft in Korea am 19. Februar 1965 äußerte der japanische Außenminister Shiina dementsprechend „tiefes Insichgehen“ angesichts der „bedauerlichen Tatsache, daß es in der langen Geschichte beider Länder eine unglückliche Periode gab"17. Die dunkle, kaum als wirkliche Übernahme historischer Verantwortung zu bewertende Aussage war freilich das Äußerste, wozu sich Shiina im Einklang mit der öffentlichen Meinung in Japan vor der Unterzeichnung des Grundlagenvertrags mit Korea im Juni 1965 verstehen mochte $^{18}$. Jede weitergehende, unzweideutige Entschuldigung wäre als „übertrieben“, ja als „Erniedrigung“ empfunden worden ${ }^{19}$. So zielte die Kritik der (sozialistischen) Opposition denn auch eher darauf ab, daß der Vertrag mit Korea die nationalen Interessen Japans nicht hinreichend berücksichtige ${ }^{20}$.

Einwände wie die eines bedeutenden Historikers und Korea-Experten, der das Fehlen oppositioneller Kritik an der Behandlung der Wiedergutmachungsfrage beklagte, blieben die Stimmen einer verschwindenden Minderheit. Neben wenigen Intellektuellen hatte sich vor allem auch der bekannte japanische Christenführer Kagawa Toyohiko schon früh, 1955, für ein Be-

16 Zit. nach ebd., S. 70.

17 Fuhrt, Erzwungene Reue, S. 70 f.

18 Zum Inhalt des Vertrags Paik, Korea und Japan, S. 272-280.

19 Fuhrt, Erzwungene Reue, S. 70 f.

20 Lee, Japan and Korea, S. 51, sowie Fuhrt, Erzwungene Reue, S. 77. 
kenntnis zur historischen Schuld ausgesprochen. In einem offenen Brief an den südkoreanischen Präsidenten schrieb Kagawa: „So wie Saulus versuchte, David zu töten, quälten die Japaner Eure Exzellenz und unterdrückten Euer Volk. Im Namen Christi entschuldige ich mich bei Eurer Exzellenz und bitte um Vergebung, indem ich an Euer christliches Gewissen appelliere. Vergeben Sie, so wie der Herr seinen Feinden am Kreuz vergeben hat, und schaffen Sie dauerhaften Frieden zwischen Großkorea und Japan." 21

Mußte ein derartiger, in christlicher Schuldkultur wurzelnder Aufruf seine Wirkung auf die Masse des Volkes verfehlen, weil in einer Schamkultur im Sinne Ruth Benedicts keine Rezeptoren hierfür ausgebildet waren oder lag dies schlicht am reinen historischen Gewissen der Japaner? Jedenfalls ist für den westlichen Analytiker von „Vergangenheitsbewältigung“ ein so bescheidenes Ausmaß nationaler historischer Selbstkritik schwer zu fassen. Insbesondere gilt dies auch für die arglos anmutende Art und Weise, mit der offizielle Darstellungen des Tokioter Finanz- und Außenministeriums später auf die Genesis der Entschädigungsabkommen zurückblickten. Japan hätte, so hieß es, Reparationszahlungen und Verträge über ökonomische Zusammenarbeit „ohne Gegenleistung hervorragend als Sprungbrett bei seiner wirtschaftlichen Expansion nach Südostasien nutzen“ können. Es sei günstig gewesen, sich „durch die Lieferung von schwer exportierbaren Fabrikanlagen und bis dahin nicht exportierten Kapitalgütern als Reparationen ,gute Bekannte' zu schaffen ...".22

Vergleicht man die von Japan geleistete „Wiedergutmachung“ mit der Entwicklung in der Bundesrepublik ${ }^{23}$, so fällt als erstes auf, daß die Alliierten, allen voran die Amerikaner - anders als gegenüber Tokio in San Francisco - bereits bis zum Ende der Besatzungszeit wichtige Pflöcke auf diesem Feld eingeschlagen hatten. Dies gilt zum einen für das Militärregierungsgesetz vom November 1947, das die Rückerstattung von durch die Arisierung geraubten Vermögenswerten vorsah. Es gilt zudem für das Entschädigungsgesetz, das noch vor Gründung der Bundesrepublik im April 1949 von der US-Regierung im Wissen darum durchgesetzt wurde, daß sich ein deutscher Gesetzgeber schwerer damit tun würde, die im Rückerstattungsgesetz noch nicht geregelten Schäden vor allem an Leben, Gesundheit und beruflichem Fortkommen aufzunehmen. Da das Gesetz nur die Entschädigung Deutscher umfaßte, die aus rassischen, religiösen oder politischen Gründen verfolgt worden waren, schloß es zahlreiche ausländische Opfergruppen in den nach 1945 zum sowjetischen Machtbereich zählenden Ländern von Entschädigung aus, vor allem auch die zahlreichen europäischen Juden ohne

21 Zit. nach Olson, Japan in Postwar Asia, S. 110.

22 Zit. nach Tanaka, Japans Nachkriegsverantwortung, S. 400.

23 Hierzu grundlegend Goschler, Wiedergutmachung; Herbst/Goschler (Hg.), Wiedergutmachung in der Bundesrepublik, und zusammenfassend vor allem Hockerts, Wiedergutmachung. 
deutsche Staatsbürgerschaft. Nach Verhandlungen mit der Claims Conference und dem Staat Israel verpflichtete sich die Bundesregierung allerdings bereits am 10. September 1952 im Luxemburger Abkommen ${ }^{24}$ zu Globalentschädigungen für Israel in Höhe von drei Milliarden DM und (in zwei Protokollen) zu einer Globalentschädigung für in der Diaspora lebende jüdische Verfolgte des Nationalsozialismus in Höhe von 450 Millionen DM sowie zu Verbesserungen der bestehenden Entschädigungsgesetze, die zu einer ganzen Reihe von problematischen Einzelfallentscheidungen geführt hatten.

Auf der Grundlage des - im Juli 1957 dann novellierten - Bundesrückerstattungsgesetzes wurden bis Mitte der 1980er Jahre sieben bis siebeneinhalb Milliarden DM ausbezahlt. Entsprechend der weiteren im Luxemburger Abkommen eingegangenen Verpflichtungen leistete die Bundesrepublik bis Ende der 1990er Jahre schließlich Zahlungen von über 100 Milliarden DM, $80 \%$ davon an jüdische Holocaust-Überlebende, von denen die Hälfte in Israel lebte. Einige Jahre nach Abschluß des Luxemburger Vertrages begannen auch Verhandlungen über Globalabkommen mit elf westeuropäischen Staaten, die mit einem Entschädigungsgesamtvolumen von ca. einer Milliarde DM zu Gunsten der sogenannten „Westverfolgten“ abgeschlossen wurden. Noch vor der Wiedervereinigung Deutschlands und Europas konnte zudem zumindest mit Polen im Rahmen der Entspannungspolitik 1975 - ohne Anerkennung eines Rechtsanspruchs - eine gewisse Wiedergutmachungsleistung (ein Wirtschaftskredit und ein Rentenabkommen in Höhe von 1 bzw. 1,3 Mrd DM) vereinbart werden ${ }^{25}$.

Nach vorsichtigen Schätzungen addieren sich die Entschädigungszahlungen der Bundesrepublik auf einen Betrag, der die japanischen Ziffern um etwa das Zehnfache übersteigt ${ }^{26}$. Noch größer ist der Unterschied, wenn man die Relationen zwischen diesen Leistungen und der Versorgung eigener Kriegsopfer in den Blick nimmt. Danach hat Tokio zur Entschädigung japanischer Opfer etwa vierzigmal so viel Geld bereitgestellt wie für ausländische; die Kriegsopferversorgung in der Bundesrepublik überstieg das Volumen der „Wiedergutmachung“ dagegen nur ca. viermal27. So eindeutig der numerische Befund ist, so behutsam sollte man ihn - gerade aus deutscher Sicht - angesichts der Tatsache beurteilen, daß auch die Verbrechensbilanz des Nationalsozialismus die des Ultranationalismus noch überstiegen hatte

${ }^{24}$ Hierzu jetzt eingehend Hansen, Aus dem Schatten der Katastrophe, S. 155-366, und Jelinek, Deutschland und Israel, S. 161-250.

25 Vgl. auch zu den Zahlenangaben Reichel, Vergangenheitsbewältigung, S. 74-88, 93.

26 Ida, Strafverfolgung, S. 111.

27 Im übrigen nicht, weil das Versorgungsrecht für die Kriegsopfer günstiger gewesen wäre, sondern wegen der viel größeren Zahl der Anspruchsberechtigten. Auch wenn man den Lastenausgleich zu Gunsten der Heimatvertriebenen hinzunimmt, der etwa 140 Milliarden Mark betrug, verändern sich die Relationen nicht wesentlich. Vgl. hierzu Hockerts, Wiedergutmachung, S. 214, sowie Ida, Strafverfolgung, S. 111. 
und daß die bundesdeutsche „Wiedergutmachung“ lange jene große, im Artikel 5 des Londoner Schuldenabkommens 1953 begründete Lücke aufwies, wonach „aus dem Zweiten Weltkrieg herrührende Forderungen von Staaten, die sich mit Deutschland im Kriegszustand befanden ... und von Staatsangehörigen dieser Staaten" prinzipiell erst im Rahmen von Verhandlungen über einen Friedensvertrag, mithin ad calendas graecas, geprüft werden sollten $^{28}$. Von den Westverfolgten und wenigen anderen Ausnahmen abgesehen geschah dies tatsächlich erst nach dem Ende des Ost-West-Konflikts in den 1990er Jahren, als es für viele Überlebende des nationalsozialistischen Terrors bereits zu spät war. Der lang anhaltende Ausschluß zahlloser NS-Verfolgter von der Entschädigung ausgerechnet in den ehemaligen Zentren des rassenideologischen Vernichtungskriegs im Osten weist somit durchaus Parallelen zum Schicksal der größten Opfergruppe des japanischen Ultranationalismus auf: den Millionen von der „Dreistrahlentaktik“ geschädigten Chinesen.

Mit der gebotenen Vorsicht vor falschem Aufrechnen lassen sich an der japanischen und bundesdeutschen Wiedergutmachungspolitik aber doch gesellschaftliche Trends erinnerungskultureller Art ablesen: Die Opferzahlen des japanischen Kriegsregimes waren jedenfalls nicht so extrem niedriger gewesen und nicht so eindeutig "nur“ herkömmlichen Kriegsverbrechen geschuldet, daß dies allein die massiv divergierenden Entschädigungszahlungen erklären könnte.

Die Diskrepanz auf der materiellen Seite der „Wiedergutmachung“ spiegelt vielmehr auch einen unterschiedlichen ideellen Zugang zu dieser Aufgabc wider, wie ein vergleichender Blick auf die koreanisch-japanischen und die deutsch-jüdischen bzw. -israelischen Beziehungen nach 1949/52 zeigt. Vor 1945 war das Verhältnis in beiden Fällen von, wenn auch im einzelnen unterschiedlich motivierten Ressentiments der deutschen bzw. japanischen Bevölkerungsmehrheit gegenüber der jüdischen bzw. koreanischen Minderheit im eigenen Staat geprägt. Wie wenig sich in Japan noch lange nach dem Krieg hieran änderte, ist bereits deutlich geworden. Um so bemerkenswerter mutet vor diesem Hintergrund der Einstellungswandel in (West-) Deutschland an, auch wenn er sich nur zögerlich von der Mehrheit der politischen Klasse auf die Bevölkerung selbst übertrug. Aufschlußreich für diesen Sachverhalt sind Inhalt und Rezeption der historischen Regierungserklärung Adenauers zur Haltung der Bundesrepublik gegenüber den Juden am 27. September 1951.

Die Regierung und die große Mehrheit des Volkes, so sagte der Kanzler, seien sich "des unermeßlichen Leides bewußt, das in der Zeit des Nationalsozialismus über die Juden ... gebracht wurde" 29 . Die im Namen des deut-

28 Hockerts, Wiedergutmachung, S. 191.

29 Keesings Archiv der Gegenwart, 27. September 1951, S. 3135. Da Adenauer in Wirklichkeit 
schen Volkes begangenen unsagbaren Verbrechen verpflichteten zur Wiedergutmachung, wenn auch in den Grenzen der wirtschaftlichen Leistungsfähigkeit. Adenauer stieß mit seiner historischen Grundsatzerklärung auf große Einigkeit im Plenum. Die Fraktionen von CDU/CSU und SPD, aber auch die damals oft noch weit nach rechts gerichteten FDP- und DP-Abgeordneten erhoben sich demonstrativ von den Sitzen. Und in der Öffentlichkeit fiel die Reaktion ähnlich aus. Es gehe nicht um Diplomatie, so meinte etwa die Süddeutsche Zeitung, „sondern um einen urmenschlichen moralischen Akt, nämlich um das Bemühen, die Schande wieder abzuwaschen, die auf den deutschen Namen gehäuft worden ist ...".30 Selbst diejenigen in den Regierungsparteien, die Adenauer nicht folgten und stärker fiskalische Bedenken gegen das Luxemburger Abkommen trugen, zogen das Ausmaß der nationalsozialistischen Judenvernichtung doch keineswegs in Zweifel. Adenauers Regierungserklärung ist teilweise „unterkühlte Distanz hinsichtlich des Leidens der Juden“ vorgehalten worden; sie habe „mehr der Exkulpierung der überwiegenden Mehrheit des deutschen Volkes als dem konkreten Bekenntnis einer Schuld oder Verantwortung “ gedient ${ }^{31}$, habe die Verstrikkungen der Gesellschaft in das System der Judenvernichtung oder zumindest ihre Gleichgültigkeit nicht hinreichend klar benannt ${ }^{32}$, habe nicht von „Völkermord“, sondern nur von „unsagbaren Verbrechen" gesprochen ${ }^{33}$. Doch obwohl diese Kritik auf der einen Seite zutrifft, berücksichtigt sie andererseits nicht hinreichend, daß Adenauer um die Widerstände gegen die "Wiedergutmachung“ in den Reihen der eigenen Partei wie in der breiten Bevölkerung wußte und sie unter den Bedingungen einer postnationalsozialistischen Massendemokratie zumindest nicht noch weitgehender ignorieren konnte, als er es ohnehin schon tat.

Nach einer Allensbach-Umfrage aus dem Jahr 1949 befürwortete zwar etwas mehr als die Hälfte der Westdeutschen eine nicht näher konkretisierte „Wiedergutmachung“, doch zeigten speziellere demoskopische Erhebungen der amerikanischen Hohen Kommission, daß sie die Notwendigkeit von Leistungen an Kriegsversehrte, Hinterbliebene und Vertriebene noch deutlich höher einstuften und etwa den Anspruch auf Rückgabe unrechtmäBig erworbener jüdischer Geschäfte überwiegend verneinten ${ }^{34}$. Das Wiedergutmachungsabkommen mit Israel selbst hielt im August 1952 eine breite

wußte, wie unpopulär die „Wiedergutmachung“ war, ist diese Formulierung wohl auch als politischer Versuch zu werten, mit den Mitteln der Rhetorik noch möglichst viele der zögernden Bundesbürger für seinen Weg zu gewinnen.

30 Süddeutsche Zeitung, 28. September 1951.

31 Stern, Im Anfang war Auschwitz, S. 326.

32 So jetzt etwa auch Hansen, Aus dem Schatten der Katastrophe, S. 126. Hansen weist darauf hin (S. 129), daß in der SPD-Fraktionssitzung nach Adenauers Regierungserklärung auch bereits Schumacher die Rede als „zu lau“ kritisiert habe.

33 Reichel, Vergangenheitsbewältigung, S. 84.

34 Ebd., S. 85. 
Mehrheit für überflüssig oder zumindest in der Summe für überhöht, wobei SPD-Anhänger im übrigen sogar noch kritischer waren als die Sympathisanten der CDU ${ }^{35}$. Die geschlossene Zustimmung der SPD-Fraktion im Bundestag, ohne die Adenauer den Vertrag - angesichts der Gegner in den eigenen Reihen - nicht durch das Parlament bekommen hätte, ist insofern besonders bemerkenswert.

Die Frage stellt sich, ob eine breitere gesellschaftliche Verankerung des historischen Schuldgefühls und damit eine höhere Bereitschaft zu finanziellen Opfern im Rahmen der „Wiedergutmachung“ bereits zu diesem Zeitpunkt realistischerweise von einem Volk zu erwarten war, aus dessen Mitte heraus sich wenige Jahre vorher die Täter - und Dutzende Millionen „Mitläufer“ rekrutiert hatten? Die Frage dahingehend zu beantworten, daß die „Wiedergutmachung" nur "das Werk eines Elitenkartells gegen die Volksmehrheit" war - und sein konnte -, würde die Problematik indes verkürzen. „Vorsichtiger und wohl richtiger ist die Formulierung: obne die Volksmehrheit“36. Denn so unpopulär die „Wiedergutmachung“ auch war, eine politisch bedeutsame Protestbewegung - in der wählenden Bürgerschaft - formierte sich gegen sie nicht. Vielmehr gelang es z.B. einem der christdemokratischen Vorkämpfer der Entschädigungsgesetze, Franz Böhm, sogar seinen schwierigen Frankfurter Wahlkreis 1953 wie auch 1957 direkt zu gewinnen ${ }^{37}$.

Auch die Stärke antisemitischer Einstellungen in der Bevölkerung nahm kontinuierlich ab. Auf die Frage: „Würden Sie sagen, es wäre besser [ist für Deutschland besser], keine Juden im Land zu haben?", antworteten 1952 noch 37\% „besser“; 1956 äußerten sich 26\% in diesem Sinne, 1963 war ihr Anteil auf $18 \%$ gesunken, um bis 1983 weiter auf $9 \%$ zu fallen ${ }^{38}$. Allerdings wird man diese Zahlen nicht ausschließlich auf eine wirkliche Einstellungsänderung, sei es individueller, sei es generationeller Art, zurückführen dürfen, sondern sie auch damit in Verbindung zu bringen haben, ,daß die öffentlich-politische Tabuisierung nur die Äußerung (Hervorhebung M.K.) antisemitischer Einstellungen ... verhindert" 39 , ohne wirklich ein schlagender Beweis für ihre Überwindung zu sein. Das Entstehen dieser Kommunikationslatenz war eine vielleicht unausweichliche Nebenwirkung jener parteiübergreifenden Entschlossenheit der bundesdeutschen classe politique zum „Frieden mit Israel“40 und zur entschiedenen Bekämpfung periodisch

35 44\% der SPD- und 37\% der CDU-Anhänger hielten das Abkommen für überflüssig; die Summe für zu hoch erachteten $27 \%$ der SPD- und $28 \%$ der CDU-Sympathisanten. Vgl.

Bergmann, Antisemitismus in öffentlichen Konflikten, S. 181.

36 Hockerts, Wiedergutmachung, S. 186.

37 Ebd.

38 Bergmann, Sind die Deutschen antisemitisch?, S. 115.

39 Ebd., S. 112.

$40 \mathrm{Zu}$ der gleichnamigen Hamburger Aktion von Rudolf Küstermeier und Erich Lüth vgl. Lüth, Ein Hamburger schwimmt gegen den Strom. 
aufflackernder antisemitischer Vorfälle in der Bundesrepublik, wie sie aus dem - nicht nur in Deutschland schwer ganz auszutrocknenden - rechtsradikalen Sumpf immer einmal wieder herauswuchsen.

Wenn man vergegenwärtigt, wie sehr es unter den Entscheidungsträgern der japanischen Koreapolitik an Protagonisten eines Versöhnungskurses mangelte, stellt sich die Frage nach den Motiven der bundesdeutschen Bemühungen um „Wiedergutmachung“ mit besonderem Nachdruck. Hat sich die Bundesrepublik damit, wie Christian Pross meint, nur aus Kalkül die Integration in das westliche Bündnis „erkauft"? 41 Oder ist das Luxemburger Abkommen, ganz im Gegenteil, „freiwillig und ohne amerikanischen Druck“ zustande gekommen, wie Michael Wolffsohn argumentiert ${ }^{42}$. Die Prioritäten der amerikanischen Politik lagen 1951/52 jedenfalls nicht beim deutsch-israelischen Abkommen, so daß die Bundesregierung einen relativ großen Handlungsspielraum besaß. Adenauer entschloß sich, „aus moralischen, außenpolitischen und außenwirtschaftlichen Motiven, hier einen möglichst starken Akzent zu setzen." 43 Auch wenn es also gar nicht unmittelbaren Drucks seitens des wichtigsten Bündnispartners bedurft hatte, ist doch evident: Das Luxemburger Vertragswerk wäre ohne die - den bundesdeutschen Akteuren sehr präsenten - außenpolitischen Rahmenbedingungen so kaum vorstellbar gewesen. Dies gilt nicht zuletzt für das von der Öffentlichkeit fast unbemerkte „Haager Protokoll Nr. 1“, das Ernst Katzenstein einen „fast revolutionären Vorgang“ genannt hat, weil damit die Claims Conference als ausländische Nichtregierungsorganisation einen vertraglich geregelten Einfluß auf die weitere innerdeutsche Entschädigungsgesetzgebung erhielt ${ }^{44}$. Wenn führende CDU-Politiker ihre Partei nachdrücklich mahnten, „die USA“ lege Wert auf das Abkommen ${ }^{45}$, so konnten sie davon ausgehen, daß das Stereotyp von den (einfluß-)reichen amerikanischen Juden in diesem Kontext von vielen mitgedacht wurde. Hatte nicht der Jüdische Weltkongreß schon im Sommer 1950 in Frankfurt - mit Bezug auf eine bundeseinheitliche Entschädigungsgesetzgebung - deutlich gemacht, die „Rückkehr Deutschlands in die Völkerfamilie“ hänge von einer befriedigenden Regelung dieser Frage $a b^{46}$ ? Und hatte US-Außenminister Dean Acheson nicht Anfang Mai 1952 eine Delegation jüdischer Amerika-

41 Pross, Wiedergutmachung, S. 292.

42 Wolffsohn, Ewige Schuld?, S. 21.

43 Hockerts, Wiedergutmachung, S. 181.

44 Ebd., S. 181.

45 So Heinrich von Brentano, zit. nach Hockerts, Wiedergutmachung, S. 180. Für Adenauer, so schreibt sein Biograph Hans-Peter Schwarz, sei die Zustimmung der jüdischen Organisationen und Zeitungen in den USA wichtiger gewesen als die Beschwerden arabischer Regierungen. Schwarz, Adenauer - Der Staatsmann, S. 65.

46 Reichel, Vergangenheitsbewältigung, S. 84. 
ner empfangen, die ihn ersuchte, Druck auf die Bundesrepublik auszuüben, um die Höhe der Wiedergutmachungszahlungen zu steigern ${ }^{47}$ ?

Wie relativ der Einfluß solch üblicher Lobby-Arbeit in der pluralistischen Demokratie Amerikas zu veranschlagen war, hat sich zumindest auch vielen bundesdeutschen Entscheidungsträgern damals nicht recht erschlossen. Deshalb wird man dem außenpolitischen Faktor „amerikanische Juden“ für das Luxemburger Abkommen mittelbar doch einiges Gewicht beizumessen haben ${ }^{48}$. Im Vergleich mit der ganz und gar unbedeutenden Rolle amerikanischer Koreaner oder Chinesen für das Kalkül japanischer Entschädigungspolitik wird dieser Befund überdeutlich. In komparativer Betrachtung zeigt sich freilich auch, daß die Geschichte der bundesdeutschen „Wiedergutmachung " nicht auf die (Fehl-)Wahrnehmung von Außendruck reduziert werden kann, sondern intrinsische Beweggründe ebenfalls stark zu berücksichtigen sind. Und das gilt nicht nur für ein kleines Häuflein führender Politiker, sondern zeigte sich auch in der klaren Stellungnahme vieler Intellektueller und Journalisten, die während der öffentlichen Debatte um das Luxemburger Abkommen Millionen bundesdeutscher Zeitungsleser und Radiohörer einmal mehr mit der Tatsache konfrontierten, daß „sechs Millionen Juden ... zerstampft, verheizt, vergiftet, erschossen, erhängt und ausgemergelt worden“ sind: „Irgendwie können wir von dem Gefühl nicht los, $\mathrm{daß}$ wir allesamt eine Art Verantwortung für jene Untaten tragen, die unter deutschem Namen begangen wurden ..."49. Charakteristische Kommentare dieser Art verdeutlichen, wie sehr die bundesdeutsche „Wiedergutmachung “ - trotz oder gerade auch wegen vieler Pannen, Skandale und moralischer Fragwürdigkeiten in der konkreten Abwicklung - eine politisch-kulturelle Dimension hatte, indem sie immer wieder Anlässe schuf, sich mit den Verbrechen der NS-Zeit - nolens volens - auseinanderzusetzen. In diesem erinnerungskulturellen Bereich fällt der Unterschied zu Japan fast noch mehr ins Auge als beim schwer zu vergleichenden finanziellen Volumen der „Wiedergutmachung“50. Denn für die Prozesse der „Vergangenheitsbewäl-

47 Trimbur, L'influence américaine, S. 203.

48 „Druck aus den USA“ spielte auch später immer wieder einmal eine Rolle; erwähnt seien nur dic von der Bundesregierung im Juni 1960 - mitten in der zweiten Berlin-Krise - beschlossenen Beihilfen für osteuropäische Opfer pseudomedizinischer KZ-Experimente, nachdem der USA-Besuch von 35 Polinnen, die das KZ Ravensbrück überlebt hatten, in der amerikanischen Öffentlichkeit hohe Wellen geschlagen hatte. Vgl. Hockerts, Wiedergutmachung, S. 194.

49 Ruhr-Nachrichten, 28. September 1951.

50 Wer den Komplex der „Wiedergutmachung“ im Rahmen einer Gesamtbewertung der „Vergangenheitsbewältigung" übergeht, kann ein vollständiges Bild der Problematik schwerlich zeichnen. Dies gilt etwa für Norbert Frei, der davon absieht, die „Wiedergutmachung“ auch nur in seine resümicrenden Überlegungen angemessen einzuordnen und - auch aufgrund dieser nicht in den Argumentationsgang passenden Auslassung - zu cinem arg einseitigen Urteil über die „vergangenheitspolitisch, langen fünfziger Jahre“ " kommt. Vgl. Frei, Vergangenheitspolitik, S. 397-406, Zitat S. 406. 
tigung " in beiden Ländern zeitigte es erhebliche Folgen, daß Parlamente und Regierungen der Bundesrepublik qua Entschädigungsgesetzgebung die kriminelle Natur des Nationalsozialismus schon früh in den 1950er Jahren gewissermaßen amtlich konstatierten und von allen seriösen Medien dabei unterstützt wurden, während ähnlich klare offizielle Verdikte über den Ultranationalismus im Rahmen der stark wirtschaftlich orientierten japanischen Entschädigungspolitik nicht nur ausblieben, sondern statt dessen lange sogar Argumente gegen die moralische Berechtigung bzw. Notwendigkeit von „Wiedergutmachung“ überhaupt den öffentlichen Diskurs beherrschten. 